ISSN: 1982-1263

\title{
Pannus do Pastor Alemão: revisão de literatura
}

\author{
Aline Maria Vasconcelos Lima ${ }^{1 *} \&$ Júlia Harger De Sousa ${ }^{2}$ \\ ${ }^{I}$ M.V., Dra., Professora da Escola de Veterinária e Zootecnia da Universidade Federal de Goiás. \\ ${ }^{2}$ Aluna de graduação em Medicina Veterinária na Escola de Veterinária e Zootecnia da Universidade Federal \\ de Goiás. \\ "Autor para correspondência, E-mail: alinevetufg@hotmail.com
}

RESUMO. O pannus é uma doença ocular que acomete cães, principalmente Pastores Alemães, caracterizada pela progressiva proliferação fibrovascular na córnea. A etiopatogenia ainda não está definitivamente determinada, contudo acredita-se ser uma condição imunomediada. O diagnóstico é estabelecido por meio dos achados clínicos, citológicos e pela resposta terapêutica, com uso de anti-inflamatórios e imunossupressores tópicos. Deve-se diferenciar o pannus de doenças como a ceratoconjuntivite seca e outras ceratites crônicas, e também de algumas neoplasias corneanas. Este trabalho objetivou realizar uma revisão de literatura sobre o pannus, abordando os aspectos clínicos, epidemiológicos, fisiopatológicos e terapêuticos desta enfermidade.

Palavras chave: Córnea, inflamação, oftalmologia.

\section{German Shepherd pannus: literatura review}

\begin{abstract}
The pannus is an eye disease that affects dogs, mostly German Shepherds, characterized by progressive proliferation of fibrovascular tissue in the cornea. The pathogenesis is not yet definitively determined, but is believed to be an autoimmune condition. The diagnosis is established by clinical, cytological findings and the therapeutic response with use of anti-inflammatory and immunosuppressive topics. One must differentiate pannus from diseases such as keratoconjunctivitis sicca and other chronic keratitis, and also some corneal neoplasia. This study aimed to conduct a literature review on the pannus, addressing the clinical, epidemiological, pathophysiological and therapeutic aspects of this disease.
\end{abstract}

Keywords: ophthalmology, corneal, inflammation.

\section{Introdução}

O pannus do Pastor Alemão, também conhecido como síndrome de Überreiter, pannus degenerativo e ceratite superficial crônica, é uma doença inflamatória que acomete a córnea de cães, caracterizada por uma proliferação fibrovascular progressiva no estroma corneano anterior (Bedford \& Lonstaffe, 1979; Slatter et al., 1997; Gilger, 2007). A primeira descrição foi de um caso isolado em 1913, sendo que relatos mais detalhados da enfermidade não foram feitos até 1959, quando Überreiter descreveu a doença em 69 cães (Slatter et al., 1997).

O pannus foi descrito em diversas raças caninas, como na Akita, Pastor Australiano, Pastor Alemão, Border Collie, Bull Mastiff,
Collie, Dachshund, Dálmata, Golden Retriever, Greyhound, Labrador, Poodle, Pastor de Shetland, Husk Siberiano e Weimarener (Bedford \& Lonstaffe, 1979; Eichenbaum et al., 1986; Chavkin et al., 1994; Slatter et al., 1997). Entretanto, a grande maioria dos casos foi descrita no Pastor Alemão, o que sugere uma condição hereditária da doença nesta raça (Andrew, 2008). Os Greyhounds tendem a ser acometidos com menos de dois anos, contudo exibem lesões relativamente moderadas (Gilger, 2007). Alguns autores observaram maior prevalência da doença em fêmeas e outros autores, em machos; contudo acredita-se não haver predileção sexual para o pannus (Bedford \& Lonstaffe, 1979; Eichenbaum et al., 1986; 
Chavkin et al., 1994; Slatter et al., 1997; Jokinen et al., 2011).

A incidência e a severidade da doença foram relacionadas a cães que habitavam em elevadas altitudes (Gilger, 2007). Em regiões com menores altitudes, a doença tende a responder mais favoravelmente à terapia menos intensa, quando se compara com a resposta à terapia em animais que vivem em maiores altitudes. Acredita-se, portanto, que a exposição aos raios ultravioleta pode exercer um papel na patogênese do pannus (Williams, 2005; Gilger, 2007; Andrew et al., 2008; Denk et al., 2011; Jokinen et al., 2011).

A maioria dos cães apresenta o pannus entre três e seis anos de idade. A idade de início da doença e a raça do cão podem ser parâmetros prognósticos para a enfermidade. Em Pastores Alemães de um a cinco anos, a doença tende a progredir rápida e severamente. Em animais de meia idade, as lesões parecem ser menos severas e evoluem lentamente (Gilger, 2007).

O pannus geralmente se manifesta na córnea perilímbica temporal inferior, onde se observa uma proliferação fibrosa rósea bem vascularizada (Figura 1). Com a evolução da doença, a placa fibrovascular se propaga para o centro da córnea e acomete também o limbo nasal, sendo potencialmente causadora de cegueira (Williams, 1999; Williams, 2005; Gilger, 2007; Andrew et al., 2008; Balicki \& Trbolova, 2010; Jokinen et al., 2011). Inicialmente as lesões podem ser unilaterais, mas na maioria dos casos são bilaterais e frequentemente assimétricas (Bedford \& Lonstaffe, 1979; Williams, 2005; Balicki \& Trbolova, 2010). Uma linha cristalina ou pequenos pontos brancos frequentemente são visibilizados no estroma corneano claro, avançando 1 a $2 \mathrm{~mm}$ da borda da lesão. Tais pontos consistem em infiltrado celular inicial associado a discreto edema corneano (Williams, 1999; Williams, 2005; Gilger, 2007; Andrew et al., 2008; Balicki \& Trbolova, 2010).

Assim como nas demais doenças corneanas que envolvem vascularização superficial, pode ocorrer a invasão da córnea por pigmentos melanóticos limbais. A porção superior da córnea usualmente permanece inalterada, até que a doença atinja seu estágio tardio, quando toda a córnea pode se tornar vascularizada e pigmentada. Alguns cães com pannus também desenvolvem um espessamento e pigmentação da superfície palpebral da terceira pálpebra e pigmentação da conjuntiva bulbar adjacente à lesão (Bedford \& Lonstaffe, 1979; Eichenbaum et al., 1986; Gilger, 2007; Andrew et al., 2008).

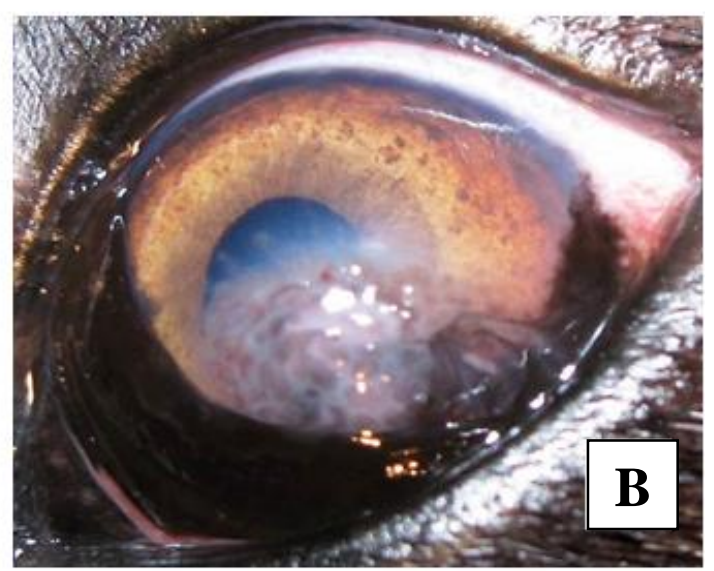

Figura 1 - Ceratite superficial crônica em cão (A) Olho esquerdo com proliferação fibrovascular do quadrante lateral inferior em direção à córnea central. Pigmentação em conjuntiva bulbar, limbo e córnea perilímbica. Notar a presença de linhas brancas e pequenos pontos brancos no estroma corneano limpo, a um milímetro da margem da lesão. (B) Olho direito com proliferação de tecido fibrovascular do quadrante lateral inferior em direção à córnea central. Pigmentação em conjuntiva bulbar, limbo e córnea perilímbica.

\section{Características histopatológicas}

À histopatologia, o pannus aparece como uma vascularização corneana superficial, com infiltração de fibroblastos e fibrócitos, e elevada densidade de linfócitos e plasmócitos no estroma corneano anterior. Poucos histiócitos e neutrófilos também podem ser visibilizados, 
estando, este último tipo celular, associado ao desenvolvimento da vascularização corneana (Williams, 1999; Andrew et al., 2008).

Os linfócitos infiltrados são predominantemente CD4+, o que sugere uma patogênese imunomediada para a doença. Foi evidenciado um número significativo de linfócitos CD4+ produtores de INF-gamma na córnea de cães com pannus (Williams, 1999). Imunoglobulinas foram identificadas no estroma conjuntival superficial próximo ao limbo e, por vezes, no estroma corneano superficial. Também se observou um aumento de GAGs sulfatados, o que pode ser resultado da atividade linfocitária na matriz extracelular (Andrew et al., 2008).

Pode ser observada hiperplasia moderada do epitélio corneano e um espessamento da membrana basal epitelial, bem como a presença de células pigmentadas (melanócitos). A pigmentação comumente acompanha o infiltrado fibrovascular inflamatório que invade o estroma (Andrew et al., 2008).

\section{Etiopatogenia}

A etiologia do pannus ainda não foi definitivamente determinada, contudo acredita-se ser uma condição imunomediada, baseando-se nas características clínicas, histopatológicas, imunológicas e na resposta ao tratamento tópico com corticosteroides e imunossupressores (Andrew et al., 2008).

A base imunomediada da doença é reforçada pela observação imunohistológica das lesões, caracterizadas pelo aumento do número e da degranulação mastocitária, pelo aumento de imunoglobulinas em sítios adjacentes e, principalmente, pela intensa infiltração de linfócitos T CD4+ secretores de IFN-gamma (Nell et al., 2005; Williams, 2005; Andrew et al., 2008). A natureza imunomediada também se reflete quando se observa a expressão exuberante de MHC II em células do estroma e epitélio da córnea de cães com pannus. Foi demonstrado que o IFN gamma, produzido pelos linfócitos que migram para a córnea acometida, é responsável pela expressão exuberante de MHCII pelas células corneanas (Williams, 2005).

Acredita-se que mesmo que a expressão de MHC II não seja definitiva para a gênese do pannus, ela exerce uma função importante em prolongar o processo inflamatório, e também pode permitir o desenvolvimento de uma reação autoimune aos antígenos normalmente presentes na córnea canina (Williams, 2005). De fato, uma resposta de hipersensibilidade às proteínas corneanas foi encontrada em cães com pannus (Nell et al., 2005). Um halotipo DLA específico foi associado com o pannus e observou-se que cães homozigotos para este locus genético possuem um aumento do risco de desenvolver o pannus (Jokinen et al., 2011).

Acredita-se que a exposição excessiva aos raios ultravioletas (UV) poderia engatilhar $\mathrm{o}$ pannus em indivíduos predispostos, por meio da alteração da antigenicidade da córnea, resultando numa lesão imunomediado ao tecido (Nell et al., 2005). A luz UV possui diferentes efeitos na modulação de moléculas imunorregulatórias, como a IL-6, TNF alfa e INF gamma (Denk et al., 2011). A exposição de células epiteliais aos raios UV também resulta na produção e liberação de citocinas proinflamatórias e de metaloproteinases (MMPs), substâncias que possuem um papel na etiologia de doenças inflamatórias, como o pannus (Chandler et al., 2008)

Uma superexpressão de MMPs na córnea de cães com pannus induzida pela radiação UV pode estar associada às alterações corneanas (Chandler et al., 2008; Denk et al., 2011). As células inflamatórias, incluindo macrófagos, neutrófilos e linfócitos T também secretam MMPs, o que aumenta a migração celular através do tecido e facilita a vascularização corneana. Ademais, MMPs preexistentes sequestradas na matriz extracelular podem ser ativadas por espécies reativas de oxigênio produzidas por macrófagos ativados pela radiação UV ou mesmo por células epiteliais expostas a estes raios. Portanto, os raios ultravioletas podem contribuir para o aumento da atividade da MMPs locais tanto por ação direta sobre as células epiteliais quanto por aumentar o influxo de células inflamatórias (Chandler et al., 2008).

A investigação de doenças infecciosas concorrentes, como a Clamidiose, não demonstrou, até o momento, a sua associação com o pannus. A observação de inclusões citoplasmáticas cilíndricas nos fibroblastos corneanos, nas células endoteliais, nos macrófagos e nas células do trabeculado de cães com pannus sugeriram uma infecção viral, mas nada definitivo foi comprovado (Nell et al., 2005; Gilger, 2007). 


\section{Diagnóstico}

Como o pannus é uma ceratopatia não ulcerativa e não dolorosa, os proprietários geralmente não observam a alteração até que se desenvolva importante proliferação tecidual ou pigmentação corneana ${ }^{6}$. O diagnóstico do pannus baseia-se nos achados clínicos, na predisposição racial e na reposta ao tratamento com imunossupressores tópicos (Gilger, 2007; Andrew et al., 2008).

A doença deve ser diferenciada da ceratoconjuntivite seca, da granulação e pigmentação corneanas secundárias às ceratites ulcerativas crônicas ou às doenças palpebrais, e também da neoplasia corneana (Gilger, 2007; Andrew et al., 2008). O teste lacrimal de Schirmer é recomendado para descartar a ceratoconjuntivite seca. A coloração com fluoresceína também é recomendada antes do início da terapia, já que a presença de ulceração requer mudança no plano terapêutico (Andrew et al., 2008).

O exame citológico pode ser realizado para a confirmação diagnóstica do pannus, evidenciando um grande número de plasmócitos, linfócitos e mastócitos (Ollivier et al, 2007; Andrew et al., 2008; Powell \& Gould, 2014). A citologia também é útil para o diagnóstico diferencial do carcinoma de células escamosas (CCE), neoplasia que frequentemente pode ser confundida com o pannus (Ollivier et al, 2007; Busse et al., 2008; Powell \& Gould, 2014). A raça acometida, a posição e aparência da lesão, e a resposta à terapia imunomoduladora também auxiliam na diferenciação do pannus e do CCE (Busse et al., 2008).

Todavia, atenção deve ser dirigida à ocorrência concomitante do pannus e do CCE. Foi constatado que cães acometidos pelo pannus apresentam dois fatores predisponentes para o desenvolvimento tumoral: a inflamação crônica e a terapia imunossupressora (Dreyfus et al., 2011). A associação entre inflamação crônica e uso de imunossupressores com o desenvolvimento de neoplasias está bem estabelecida na espécie humana. Dentre os fatores que poderiam contribuir com o desenvolvimento do CEE em pacientes com pannus, apontou-se o dano celular pela radiação UV e as alterações dos sinais de apoptose celular, da função de supressão de tumor e da vigilância imune contra os tumores sob a influência da terapia imunossupressora (Dreyfus et al., 2011).

\section{Tratamento}

O pannus geralmente pode ser controlado por uma variedade de métodos medicamentosos e cirúrgicos, mas não pode ser curado. $\mathrm{O}$ proprietário deve ser informado sobre a necessidade de terapia durante toda a vida do cão para controlar essa doença, e sobre a severidade e o prognóstico do pannus, que dependem de vários fatores, incluindo a idade do aparecimento da enfermidade, a localização geográfica e a altitude da região onde vive o paciente (Gilger, 2007).

\section{a) Corticosteroides}

Admite-se como terapia inicial o uso de colírios ou pomadas de corticosteroides à base de dexametasona a $0,1 \%$ ou de prednisona a $1 \%$, na frequência de três a quatro vezes ao dia, durante três a quatro semanas, seguida pela redução terapêutica (Gilger, 2007). Em alguns casos, entretanto, a injeção subconjuntival de corticosteroides, em adição à terapia tópica, pode ser necessária para o controle da doença (Andrew, 2008). A associação da dexametasona com DMSO 50\% tópicos mostrou-se mais efetiva que a terapia isolada com dexametasona (Balicki, 2007).

Os efeitos deletérios induzidos por colírios esteroides observados em humanos, como o glaucoma e a catarata, parecem não ocorrer em cães. Entretanto, supressão adrenocortical subclínica pode ser evidenciada após o uso prolongado de colírio corticosteroide no cão. Adicionalmente, os animais medicados por um longo tempo com corticosteroides necessitam de acompanhamento devido ao risco de infecção ocular ou ulceração corneana (Gilger, 2007).

\section{b) Imunomoduladores}

Os fármacos imunomoduladores são altamente lipofílicos e passam rapidamente através da membrana celular, para, no citoplasma, atuar no sistema mensageiro de ativação de células $T$. Os imunomoduladores irão exercer seus efeitos quando se ligarem a receptores ou a proteínas citoplasmáticas denominadas imunofilinas. $\mathrm{O}$ complexo imunomodulador-proteína interage com substratos adicionais, resultando numa atividade imunossupressora (Moore, 2004). As moléculas imunomoduladoras já estudadas para o tratamento do pannus são a Ciclosporina $\mathrm{A}$, o Tacrolimus e o Pimecrolimus. 
A ciclosporina A (CsA) é um undecapeptideo cíclico neutro, lipofílico, isolado do fungo Tolyplocadium inflatum. Em concentrações terapêuticas, esta molécula age como um imunossupressor pela inibição de linfócitos $\mathrm{T}$, sem provocar citotoxicidade (Moore, 2004; Gilger et al., 2005; Spiess et al., 2009). A CsA age inicialmente no ciclo de ativação celular, levando à falha da transcrição de genes que codificam citocinas responsáveis pela proliferação llinfocitária, como a IL-2, IL-4 e IFN-gamma (Gilger et al., 2005).

No citoplasma dos linfócitos, a CsA liga-se à imunofilina ciclofilina. O complexo CsAciclofilina liga-se, ainda no citoplasma, a uma proteína cálcio-dependente, denominada calcineurina. A calcineurina é uma proteína que promove a passagem do Fator Nuclear de Ativação Linfocitária (NF-AT) do citoplasma para o núcleo celular, onde este fator promoverá a transcrição gênica de citocinas responsáveis pela ativação linfocitária, como a IL-2 (Gilger et al., 2005). Quando a Calcineurina liga-se ao complexo CsA-ciclofilina, este complexo bloqueia a habilidade da Calcineurina de translocar o NF-AT para o núcleo, prevenindo assim a transcrição e a expressão genica de ativadores da função linfocitária (IL-2). Além de reduzir a secreção linfocitária de IL-2, a CsA interfere com os receptores de IL-2 na superfície dos linfócitos (Moore, 2004; Gilger et al., 2005).

Outras teorias postulam que a CsA poderia interferir diretamente na ligação entre o NF-AT e o seu DNA cognato, ou que ela poderia de alguma forma reduzir a atividade das proteínas cinases transdutoras, as quais promovem sinais de cálcio para a fosforilação e ativação do NFAT. A CsA também foi apontada como capaz de afetar eosinófilos, reduzindo sua produção, e mastócitos, bloqueando a degranulação e a transcrição de genes ativadores de mastócitos (IL-3 e IL-5), além de suprimir a produção de TNF-alfa pelas células B (Moore, 2004; Gilger et al., 2005).

$\mathrm{Na}$ medicina veterinária, a CsA é usada em diversas doenças oculares imunomediadas, incluindo a ceratoconjuntivite seca, a conjuntivite plasmocítica e o pannus (Spiess et al., 2009). Este fármaco pode ser utilizado para o tratamento do pannus numa concentração de $0,2 \%$ a $2 \%$, e numa frequência de duas vezes ao dia durante três a quatro semanas. Após este período o paciente deve ser reexaminado para determinar se a redução da terapia pode ser indicada (Andrew et al., 2008). Alguns animais poderão apresentar longos períodos entre os episódios de doença ativa, enquanto que outros cães necessitarão de terapia imunomoduladora contínua para manutenção (Andrew et al., 2008).

Estudos compararam o uso de dexametasona tópica três vezes ao dia com o uso da CsA $0,2 \%$ duas vezes ao dia para o tratamento do pannus, constatando que estes fármacos possuem efetividade semelhante (Gilger, 2007; Andrew et al., 2008). Contudo, devem-se considerar os efeitos colaterais decorrentes do uso de corticosteroides tópicos no longo prazo. Em humanos, a irritação da superfície ocular secundária ao uso da CsA pode ocorrer, efeito que pode estar associado ao fármaco ou ao veículo. Sob o aspecto dos efeitos sistêmicos da CsA, existem controvérsias sobre a quantidade de fármaco absorvido após aplicação tópica e sobre as alterações na atividade de linfócitos circulantes. Em felinos com ceratite eosinofilica, não foi demonstrado nenhum efeito sistêmico deletério com o uso tópico da CsA de $0,2 \%$ a $2 \%$ em cães (Spiess et al., 2009).

O Tacrolimus é um antibiótico macrolídeo isolado do Streptomyces tsukubaensis, com propriedades imunossupressoras e antiinflamatórias. Assim como a CsA, o Tacrolimus também age na fase inicial de ativação das células $\mathrm{T}$, prevenindo a transcrição de genes ativadores de linfócitos T (IL-2, IL-3, IL-4, IFNgana, TNF alfa, GMCSF, c-myc ). Contudo, enquanto que no citoplasma das células $\mathrm{T}$ a CsA liga-se à imunofilina ciclofilina, o Tacrolimus liga-se à uma imunofilina denominada FKBP proteína (Williams, 1999; Moore, 2004; Spiess et al., 2009).

Em humanos, o Tacrolimus é uma alternativa efetiva à CsA na prevenção da rejeição aos transplantes, sendo considerado dez a 100 vezes mais potente que a CsA in vitro (Williams, 1999). Em cães acometidos pela ceratoconjuntivite seca imunomediada, o Tacrolimus mostrou-se tão efetivo quanto a CsA, sendo considerada uma alternativa quando ocorre falha na resposta ao tratamento com a CsA (Moore, 2004; Joseph et al., 2005; Hendrix et al., 2011)

O uso do Tacrolimus colírio a $0,02 \%$ três vezes ao dia no tratamento do pannus em cães proporcionou importante remissão da vascularização corneana após cinco semanas de 
terapia. Adicionalmente, nos casos em que se observou granulação da córnea, o Tacrolimus promoveu uma redução efetiva do tecido fibrovascular (Balicki \& Trbolova, 2010).

Outros estudos demonstraram boa tolerância ao uso da medicação a $0,02 \%$ em forma de pomada, sem a ocorrência de irritação ocular (Balicki \& Trbolova, 2010).

O Pimecrolimus é um derivado da ascomicina, que interfere seletivamente com a ativação de células $\mathrm{T}$ e mastócitos, e inibe a produção de citocinas inflamatórias. No nível molecular, o pimecrolimus liga-se ao receptor citoplasmático da macrofilina 12 e inibe a ação da calcineurina, assim como a CsA e o Tacrolimus. Como consequência, evita a transcrição e a liberação de citocinas inflamatórias de linfócitos $\mathrm{T}$, bem como impede a proliferação destas células. Em comparação com a CsA, o Pimecrolimus pode ter potência superior devido à sua maior permeabilidade tecidual decorrente do seu menor peso molecular (Nell et al., 2004; Nell et al., 2005; Ofri et al., 2009).

Em um estudo o Pimecrolimus a $1 \%$ foi utilizado em forma de colírio oleoso para o tratamento do pannus, três vezes ao dia, durante 36 semanas. Após duas semanas de tratamento observou-se importante regressão da vascularização corneana, a qual apresentou completa remissão em onze semanas de tratamento. Adicionalmente, observou-se regressão contínua da intensidade e da extensão da pigmentação corneana (Nell et al., 2005). Consideraram-se, neste estudo, promissores os resultados obtidos com o uso do Pimecrolimus no pannus, embora alguns animais tenham apresentado intolerância à medicação, como irritação e prurido após o uso prolongado do fármaco; e outros poucos não tenham apresentado melhora dos sinais clínicos com o uso do Pimecrolimus (Nell et al., 2005).

\section{c) Ceratectomia lamelar superficial}

Em casos severos não responsivos à terapia com corticosteroides e imunomoduladores, e nos quais a pigmentação da córnea central resultou em cegueira, terapias mais invasivas, como procedimentos cirúrgicos, podem ser requeridos. A ceratectomia lamelar superficial foi sugerida para o tratamento do pannus para casos severos, contudo a recorrência deve ser esperada e outras ceratectomias podem ser necessárias (Gilger, 2007; Andrew, 2008). Muita atenção deve ser dada ao período de tempo entre ceratectomias sucessivas, devido ao adelgaçamento corneano resultante do procedimento (Gilger, 2007).

Adicionalmente existem relatos do uso da transposição corneoescleral e do implante de ceratoprótese, em casos de pannus com opacificação da córnea central associada a não resposta ao tratamento médico (Andrew, 2008; Allgoewer et al., 2010; Isard et al., 2010).

\section{d) Radioterapia}

A radioterapia pode ser uma alternativa nos casos de pannus severo e não responsivo ao tratamento medicamentoso, associada ou não à ceratectomia superficial. As maiores fontes de radiação beta para o tratamento do pannus são os raios X e o estrôncio 90 (Sr-90). Contudo, os estudos controlados sobre o uso da radioterapia para o tratamento do pannus são escassos, e existem poucas informações sobre a dose e os efeitos colaterais, além de possuir uso restrito em cães, devido seu alto custo (Hoch et al., 2002; Grüning et al., 2011; Allgoewer \& Hoecht, 2014; Azouley, 2014).

A radiação ionizante pode exercer um efeito direto na pigmentação corneana por meio da inibição da síntese de RNA e da atividade da tirosinase na córnea. Indiretamente, a radiação também reduz a vascularização corneana, por meio da inibição da mitose das células endoteliais. O bloqueio da vascularização impede a migração de melanócitos limbais para o estroma corneano. Adicionalmente, com a regressão da vascularização, a córnea perde seu estado inflamatório ativo e retorna ao seu estado normal quiescente (Grüning et al., 2011; Allgoewer \& Hoecht, 2014; Azouley, 2014).

\section{Manejo}

Como a incidência e a severidade do pannus correlacionam-se com a altitude geográfica e com a incidência de radiação UV, presume-se que a proteção contra os raios ultravioletas pode exercer um papel adjuvante no controle da enfermidade. $\mathrm{O}$ uso de óculos escuros foi apontado como uma alternativa, contudo estes acessórios não são bem aceitos por todos os cães e os géis protetores possuem uma vida útil curta (Denk et al., 2011).

Estudos têm testado o uso de lente de contato bloqueadora de raios UV como adjuvante no tratamento do pannus, com resultados positivos. Contudo, o uso prolongado de lentes de contato 
em cães requer uma cuidadosa seleção do paciente, higienização relativamente rigorosa do material e uma boa complacência e dedicação do proprietário (Fritsche et al., 2007; Denk et al., 2011).

\section{Considerações Finais}

O pannus ainda é um desafio terapêutico para o Médico Veterinário, especialmente quando a pigmentação corneana torna-se extensa. O diagnóstico precoce é, portanto, uma ferramenta útil para que se evite a progressão da opacidade da córnea e a perda visual. Adicionalmente, as evidências de componentes genéticos envolvidos com a manifestação da enfermidade podem apontar, no futuro, para uma nova perspectiva de tratamento e prevenção do pannus.

\section{Referências}

Allgoewer, I. \& Hoecht, S. 2010. Radiotherapy for canine chronic superficial keratitis using soft X-rays $(15 \quad \mathrm{kV}) . \quad$ Veterinary Ophthalmology, 13, 2, 20-25.

Allgoewer, I.; Mclellan, G. J. \& Agarwal, S. 2010. A keratoprosthesis prototype for the dog. Veterinary Ophthalmology, 13, 1, 47-52.

Andrew, S. E. Immune-mediated canine and feline keratitis. 2008. The Veterinary Clinics of North America. Small Animal Practice, 38, 2, 269-290.

Azouley, T. 2014. Adjunctive cryotherapy for pigmentary keratitis in dogs: a study of 16 corneas. Veterinary Ophthalmology, 17, 4, 241-249.

Balicki, I. 2007. The use of dexamethasone and dimethyl sulfoxide for chronic superficial keratitis treatment in dogs. In: Annual Meeting of the European College of Veterinary Ophthalmologists, 10, 6, 323-335.

Balicki. I. \& Trbolova, A. 2010. Clinical evaluation of Tacrolimus eye drops for chronic superficial keratitis treatment in dogs. Bulletin of the Veterinary Institute in Pulawy, 54, 2, 251-258.

Bedford, P. G. C. \& Lonstaffe, J. A. 1979. Corneal pannus (chronic superficial keratitis) in the German Shepherd dog. The Veterinary Clinics of North America. Small Animal Practice, 20, 1, 41-56.

Busse, C.; Sansom, J.; Dubielzig, R. R. \& Hayes, A. 2008. Corneal squamous cell carcinoma in a Border collie: Short communication. Veterinary Ophthalmology, 11, 1, 55-58.

Chandler, H. L.; Kusewitt. D. F. \& Colitz, C. M. H. 2008. Modulation of matrix metalloproteinases by ultraviolet radiation in the canine cornea. Veterinary Ophthalmology, $11,3,135-144$.

Chavkin, M. J.; Roberts S. M. \& Salman, M.D. 1994. Risk factors for development of chronic superficial keratitis in dogs. Journal of the American Veterinary Medical Association, 204, 10, 1630-1634.

Denk, N.; Fritsche, J. \& Reese, S. 2011. The effect of UV-blocking contact lenses as a therapy for canine chronic superficial keratitis. Veterinary Ophthalmology, 14, 3, 186-194.

Dreyfus, J.; Schobert, C. S. \& Dubielzig, R. R. 2011. Superficial corneal squamous cell carcinoma occurring in dogs with chronic keratitis. Veterinary Ophthalmology, 14, 3, 161-168.

Eichenbaum, J. D.; Lavach, J. D. \& Gould, D. H. 1986. Immunohistochemical staining patterns of canine eyes affected with chronic superficial keratitis. American Journal of Veterinary Research, 47, 9, 1952-1955.

Fritsche, J.; Denk, N.; Kafarnik, C. \& Leuzinger, K. 2007. Is the long-term management of dogs with UV blocking contact lenses possible in practice? A field study. In: Annual Meeting of the European College of Veterinary Ophthalmologists, 10, 6, 323-335.

Gilger, B. C. 2007. Diseases and surgery of the canine cornea and sclera. In: Gelatt, K. N. (4 ed.) Veterinary ophthalmology, Blackell Publishing, Ames, IA, USA.

Gilger, B. C.; Michau, T. M. \& Salmon, J. H. 2005. Immune-mediated keratitis in horses: 19 cases (1998-2004). Veterinary Ophthalmology, 8, 4, 233-239.

Grüning, G. \& Allgoewer, I.; Hocht, S. 2011. Zur Strahlentherapie der Keratitis superficialis chronica mit Strontium 90. Kleintierpraxis, 46, 389-399.

Hendrix, D. V. H.; Adkins, E. A.; Ward, D. A.; Stuffle, J. \& Skorobohach, B. 2011. An Investigation Comparing the Efficacy of Topical OcularApplication of Tacrolimus and 
Cyclosporine in Dogs. Veterinary Medicine International, 2011, 1-5.

Hoch, S.; Grüning, G. \& Allgoewer, I. 2002. Die Behandlung der Keratitis chronica superficialis des Hundes mit Strontium-90. Strahlentherapie und Onkologie, 178, 99-104.

Isard, P.; Dulaurent, T. \& Regnier, A. 2010. Keratoprosthesis with retrocorneal fixation: preliminary results in dogs with corneal blindness. Veterinary Ophthalmology, 13, 5, 279-288.

Jokinen, P.; Rusanen, E. M.; Kennedy, L.J. \& Lohi, H. 2011. MHC class II risk haplotype associated with canine chronic superficial keratitis in German Shepherd dogs. Veterinary Immunology and Immunopathology, 140, 2, $37-41$.

Joseph, M.A.; Kaufman, H.E. \& Insler M. 2005. Topical tacrolimus ointment for treatment of refractory anterior segment inflammatory disorders. Cornea, 24, 417-420.

Moore, C. P. 2004. Immunomodulating agents. The Veterinary Clinics of North America. Small Animal Practice, 34, 3, 725-737.

Nell, B.; Walde, I.; Billich, A.; Vit, P. \& Meingassner, J. G. 2005. The effect of topical pimecrolimus on keratoconjunctivitis sicca and chronic superficial keratitis in dogs: results from an exploratory study. Veterinary Ophthalmology, 8, 1, 39-46.

Nell, B.; Walde, I.; Spiess, B. M.; Rueheli, M.; Vit, P.; Billich, A.;. Meingassner, J. G. \& Stuetz, A. 2004. Exploratory Study Reveals Topical Pimecrolimus Is Active Against Keratoconjunctivitis Sicca And Chronic Superficial Keratitis In Dogs. . Investigative Ophthalmology and Visual Science; 45: EAbstract 131.

Ofri, R.; Lambrou, G. N.; Allgoewer, I.; Granitz, Pena, T. M. \& Spiess, B. M. 2009. Clinical evaluation of pimecrolimus eye drops for treatment of canine keratoconjunctivitis sicca: A comparison with cyclosporine A. The Veterinary Journal, 179, 70-77.
Ollivier, F.J.; Plummer, C.E. \& Barrie, K. P. 2007. Ophthalmic examination and diagnosis part 1: the eye examination and diagnostic procedure. In: Gellat, K.N. Veterinary Ophthalmology, Blackell Publishing, Ames, Iowa, USA.

Powell, R. \& Gould, D. 2014. Cytology of the Eye and Adnexal Structures. In Dunn, J. (1 ed.) Manual of diagnostic cytology of the dog and the cat. Wiley Blackwell, Somerset, NJ, USA.

Slatter D. H, Lavach J. D. \& Severin G. A. 1977. Uberreiter's syndrome (chronic superficial keratitis) in dogs in the Rocky Mountain areaa study of 463 cases. The Veterinary Clinics of North America. Small Animal Practice, 18, $12,757-772$.

Spiess, A. K.; Sapienza, J. S. \& Mayordomo, A. 2009. Treatment of proliferative feline eosinophilic keratitis with topical $1.5 \%$ cyclosporine: 35 cases. Veterinary Ophthalmology, 12, 2, 132-137.

Williams, D. L. 1999. Histological and immunohistochemical evaluation of canine chronic superficial keratitis. Research in Veterinary Science, 67, 7, 189-193.

Williams, D. L. 2005. Major histocompatibility class II expression in the normal canine cornea and in canine chronic superficial keratitis. Veterinary Ophthalmology, 8, 6, 395-400.

\section{Recebido em Novembro 8, 2014}

Aceito em Agosto 20, 2015

License information: This is an open-access article distributed under the terms of the Creative Commons Attribution License, which permits unrestricted use, distribution, and reproduction in any medium, provided the original work is properly cited. 